\title{
Convergence of the Rotating Fluids system in a domain with rough boundaries.
}

\author{
David Gérard-Varet
}

\begin{abstract}
We consider a rotating fluid in a domain with rough horizontal boundaries. The Rossby number, kinematic viscosity and roughness are supposed of characteristic size $\varepsilon$. We prove a convergence theorem on solutions of NavierStokes Coriolis equations, as $\varepsilon$ goes to zero, in the well prepared case. We show in particular that the limit system is a two-dimensional Euler equation with a nonlinear damping term due to boundary layers. We thus generalize the results obtained on flat boundaries with the classical Ekman layers. The complete proofs can be found in [12].
\end{abstract}

\section{Introduction}

We will study in this paper Navier-Stokes Coriolis equations

$$
\begin{aligned}
\partial_{t} u+u \cdot \nabla u+\frac{\mathbf{e} \times u}{\operatorname{Ro}}+\frac{\nabla p}{\operatorname{Ro}}-\nu_{H} \Delta_{H} u-\nu_{V} \partial_{z}^{2} u & =0, \\
\operatorname{div} u & =0 .
\end{aligned}
$$

This system models the evolution of an incompressible rotating fluid, submitted to the Coriolis force $\mathrm{Ro}^{-1} \mathbf{e} \times u$ and viscous forces $-\nu_{H} \Delta_{H} u-\nu_{V} \partial_{z}^{2} u$. Vector $\mathbf{e}=(0,0,1)^{t}$ is the rotation axis, Ro is the Rossby number, $\nu_{H}$ and $\nu_{V}$ are the horizontal and vertical kinematic viscosities. With appropriate choices of these parameters, equations (1.1), (1.2) are involved in the description of many geophysical flows: we refer to [20] for a detailed physical insight. In the sequel, we will suppose that

$$
\operatorname{Ro}=\nu_{H}=\nu_{V}=\varepsilon \ll 1 .
$$

It is a geophysical scaling, notably relevant to the Earth's liquid core, for which Ro $\sim 10^{-7}$ and $\nu_{H} \sim \nu_{V} \sim 10^{-8}$ (see [9] for more).

MSC 2000:76U05, 76D05, 76D10

Keywords : Rotating Fluids, incompressible Navier-Stokes equations, boundary layers, rough domains.. 
System (1.1), (1.2) then reduces to

$$
\begin{aligned}
\partial_{t} u+u \cdot \nabla u+\frac{\mathbf{e} \times u}{\varepsilon}+\frac{\nabla p}{\varepsilon}-\varepsilon \Delta u & =0, \\
\operatorname{div} u & =0 .
\end{aligned}
$$

in a domain $\Omega^{\varepsilon}$ to be precised later on. Completed with appropriate initial data and boundary conditions, this system has global Leray solutions (see [24])

$$
u^{\varepsilon} \in L^{\infty}\left(0,+\infty ; L^{2}\right)^{3} \cap L^{2}\left(0,+\infty ; H^{1}\right)^{3} .
$$

The proof is the same as for classical Navier-Stokes equations, because the Coriolis term does not play any role in the energy estimates. It is then natural to ask about the behaviour of $u^{\varepsilon}$ as $\varepsilon$ goes to 0 .

Physically, this question has been the matter of an extensive study (cf [14, 22, 23]), outlining two features: the development of highly oscillatory waves, due to the skewsymmetric Coriolis operator, and in domains with boundaries, the development of boundary layers.

$>$ From a mathematical viewpoint, the study of such boundary layers has been limited to simple domains, namely $\Omega^{\varepsilon}=\mathbb{T}^{2} \times(0,1)$ or $\Omega^{\varepsilon}=\mathbb{R}^{2} \times(0,1)$. In this case, the basic idea is to construct an approximate solution of type

$$
u_{\text {app }}^{\varepsilon}(t, x, y, z)=u(t, x, y)+\tilde{u}\left(t, x, y, \frac{z}{\varepsilon}\right)+\bar{u}\left(t, x, y, \frac{1-z}{\varepsilon}\right)
$$

where

- $u$ is a two-dimensional interior term (i.e. $u_{3}=0$ ).

- $\tilde{u}=\tilde{u}(t, x, y, \theta)$ and $\breve{u}=\breve{u}(t, x, y, \lambda)$ are boundary layer terms (Ekman layers), solutions of a linear differential system in $\theta$ and $\lambda$ respectively.

Under appropriate assumptions on the initial data, namely well-prepared and small in $L^{\infty}, u^{\varepsilon}$ converges to $u$ in $L^{\infty}\left(0,+\infty ; L^{2}\right)^{3}$. The limit term $u$ is the solution of a damped Euler equation, with a dissipative term due to the boundary layer (the so-called "Ekman pumping"). We refer to [15] for details, and to [6], [7], [8] for similar work on close geophysical systems. Let us also mention recent refinement [21], in which the smallness assumption is relaxed. Note that if the viscous term in (1.3) is replaced by an anisotropic one, no smallness assumption is required (cf [15]). Moreover, the anisotropic diffusion allows to handle the high-frequency waves, and to obtain a convergence theorem for general initial data, as shown in articles [5, 18].

In this paper, we wish to extend such convergence results to more general and realistic domains. The question of the convergence of $u^{\varepsilon}$ for general domains $\Omega$ is still widely open. Even at a formal level, difficulties arise, with various possible boundary layer sizes (see [13] for more). As a first step, a convergence result has been obtained in [3] in the case of an anisotropic viscosity and a cylindrical geometry.

The aim of this note is to deal with rough boundaries. We will consider irregularities with characteristic size $\varepsilon$, in both horizontal and vertical directions. This is 
motivated by the study of the core-mantle boundary, as explained in [19]. Mathematically, the effect of rugosity on a flow has been widely studied in the context of wall laws: see for instance articles $[1,10,16,2]$ and references therein. Most of these papers study channel flows, for which the boundary layer correction to the limit flow (for instance Poiseuille or Couette flow) has at most amplitude $O(\varepsilon)$. In the system (1.3), (1.4) studied here, boundary layers have an amplitude $O(1)$ and modify the limit flow. Moreover, the equations involved in the construction of the approximate solution will be much more complex than in the "flat case": the differential system on the boundary layer will turn into a nonlinear PDE, and the Ekman pumping term also becomes nonlinear.

\section{The rough domain and statement of the results}

\subsection{The domain $\Omega^{\varepsilon}$}

Let us model the domain $\Omega^{\varepsilon}$ in which equations (1.3), (1.4) hold. We write

$$
\Omega^{\varepsilon}=\Omega \cup \Sigma_{1} \cup \Sigma_{2} \cup \Omega_{1}^{\varepsilon} \cup \Omega_{2}^{\varepsilon} .
$$

- $\Omega$ is the interior domain $(0,1)^{3}$.

- $\Sigma_{1}=(0,1)^{2} \times\{0\}$ and $\Sigma_{2}=(0,1)^{2} \times\{1\}$ are the interfaces.

- $\Omega_{1}^{\varepsilon}$ and $\Omega_{2}^{\varepsilon}$ are the rough layers. They are supposed to be generated by homothety and translations of "canonical cells of roughness".

More precisely, for $j=1,2$, let $\gamma_{j}$ a Lipschitz surface, $Z=\gamma_{j}(X, Y), \gamma_{j}:(0,1)^{2} \mapsto$ $[0,1)$. We assume that $\Gamma_{j}=\cup_{k \in \mathbb{Z}^{2}}\left(k+\gamma_{j}\right)$ is also a Lipschitz surface. The canonical cells of roughness are defined by

$$
\mathcal{R}_{j}=\left\{(X, Y, Z) \mid(X, Y) \in(0,1)^{2}, 1>Z>\gamma_{j}(X, Y)\right\}
$$

We then set

$$
\Omega_{1}^{\varepsilon}=\left(\bigcup_{k \in \mathbb{Z}^{2}} \varepsilon\left(\mathcal{R}_{1}+\left(k_{1}, k_{2},-1\right)\right)\right) \cap\left((0,1)^{2} \times(-\varepsilon, 0)\right) .
$$

For simplicity, we suppose that $1 / \varepsilon$ is an integer, so that $\Omega_{1}^{\varepsilon}$ consists of a large number of periodically distributed humps of characteristic length and amplitude $\varepsilon$. In the same way $\left(\mathbf{e}=(0,0,1)^{t}\right)$,

$$
\Omega_{2}^{\varepsilon}=\mathbf{e}+\left(\bigcup_{k \in \mathbb{Z}^{2}} \varepsilon\left(-\mathcal{R}_{2}+\left(k_{1}, k_{2}, 1\right)\right)\right) \cap\left((0,1)^{2} \times(1,1+\varepsilon)\right) .
$$

We note $\Gamma_{1}^{\varepsilon}$ and $\Gamma_{2}^{\varepsilon}$ the lower and upper horizontal boundaries of $\Omega^{\varepsilon}$.

In what follows, we will consider solutions of (1.3), (1.4) satisfying

$$
u^{\varepsilon} \text { 1-periodic in }(x, y), \quad u^{\varepsilon}=0 \text { at } \Gamma_{1}^{\varepsilon} \cup \Gamma_{2}^{\varepsilon} .
$$


For this boundary condition, the existence of Leray solutions is of course still valid.

Besides the global domain $\Omega^{\varepsilon}$, we also need to introduce boundary layer domains $\tilde{\omega}$ and $\bar{\omega}$ defined as follows

$$
\tilde{\omega}=\tilde{\omega}^{+} \cup \Sigma \cup \tilde{\omega}^{-}, \quad \bar{\omega}=\bar{\omega}^{+} \cup \Sigma \cup \bar{\omega}^{-}
$$

where

$$
\begin{gathered}
\tilde{\omega}^{+}=(0,1)^{2} \times \mathbb{R}^{+}, \quad \Sigma=(0,1)^{2} \times 0, \quad \tilde{\omega}^{-}=R_{1}-(0,0,1), \\
\bar{\omega}^{+}=-R_{2}+(0,0,1), \quad \bar{\omega}^{-}=(0,1)^{2} \times \mathbb{R}^{-} .
\end{gathered}
$$

We note $\tilde{\gamma}$ and $\bar{\gamma}$ the horizontal boundaries of $\tilde{\omega}$ and $\bar{\omega}$. Finally, for all positive $R$, $R_{1}$ and $R_{2}$, we note

$$
\tilde{\omega}^{R}=\tilde{\omega} \cap\{z>R\}, \quad \tilde{\omega}^{R_{1}, R_{2}}=\tilde{\Omega} \cap\left\{R_{1}<Z<R_{2}\right\} .
$$

\subsection{Statement of the results}

As usual with boundary layer problems, the study of $u^{\varepsilon}$ involves auxiliary systems.

- The first one holds in $\tilde{\omega}$ : for $\mathbf{u} \in \mathbb{R}^{2}$, we consider equations:

$$
\begin{aligned}
\mathbf{e} \times \tilde{u}+\nabla \tilde{p}+\tilde{u} \cdot \nabla \tilde{u}-\Delta \tilde{u}=\left(\begin{array}{c}
-\mathbf{u}^{+} \\
0
\end{array}\right) & \text { in } \tilde{\omega}^{-}, \\
\mathbf{e} \times \tilde{u}+\nabla \tilde{p}+\tilde{u} \cdot \nabla \tilde{u}-\Delta \tilde{u}=0 & \text { in } \tilde{\omega}^{+}, \\
\operatorname{div} \tilde{u}=0 & \text { in } \tilde{\omega}^{+} \cup \tilde{\omega}^{-}, \\
{[\tilde{u}]_{\mid \Sigma}=-\left(\begin{array}{c}
\mathbf{u} \\
0
\end{array}\right) } & \text { on } \Sigma, \\
{\left[\frac{\partial \tilde{u}}{\partial_{Z}}-\tilde{p} \mathbf{e}\right]_{\mid \Sigma}=0 } & \text { on } \Sigma, \\
\tilde{u}=0 \quad \text { on } \tilde{\gamma}, & \tilde{u} \text { 1-periodic in }(X, Y)
\end{aligned}
$$

where $\tilde{u}: \tilde{\omega} \mapsto \mathbb{R}^{3}, \tilde{p}: \tilde{\omega} \mapsto \mathbb{R}, \mathbf{e}=(0,0,1)^{t}$, and $[f]_{\mid \Sigma}=f^{+}-f^{-}$is the jump of $f$ at the interface $\Sigma$. We state

Theorem 2.1 There exists $U_{\infty} \in \mathbb{R}$, such that for all $|\mathbf{u}| \leq U_{\infty}$, (BL) has a unique variational solution $(\tilde{u}, \tilde{p})$, in the sense given in next section.

Moreover, for $R$ large enough, for all $m \geq 0,(\tilde{u}, \tilde{p}) \in H^{m}\left(\tilde{\omega}^{R}\right)^{4}$ with the estimate

$$
\|\tilde{u}\|_{H^{m}(\tilde{\omega} R)}+\|\tilde{p}\|_{H^{m}\left(\tilde{\omega}^{R}\right)} \leq C_{m} \exp (-\sigma R),
$$

where $\sigma>0$ is independent of $m, R$.

- The second one (which is the limit system) is two-dimensional. It holds in $\mathbb{T}^{2}$, i.e. $(0,1)^{2}$ with 1-periodic boundary conditions on $(x, y)$ : we consider equations

$$
\begin{aligned}
\partial_{t} \zeta+u \cdot \nabla \zeta+\operatorname{curl} P(u) & =0, \\
\zeta=\operatorname{curl} u, \operatorname{div} u & =0, \\
u_{\mid t=0} & =u_{0}
\end{aligned}
$$


where $u=u(t, x, y): \mathbb{R}^{+} \times \mathbb{T}^{2} \mapsto \mathbb{R}^{2}$, curl $u=\partial_{x} u_{2}-\partial_{y} u_{1}$. The "pumping function" $P$ is defined by $P=\tilde{P}+\bar{P}$, where $\tilde{P}(\mathbf{u})=\int_{\tilde{\omega}}\left(\begin{array}{c}\tilde{u}_{2} \\ -\tilde{u}_{1}\end{array}\right),|\mathbf{u}|<U_{\infty}, \tilde{u}$ solution of (BL), as given by theorem 2.1. Function $\bar{P}$, related to the upper boundary layer, is similar (see next section). $P(\mathbf{u})$ is a dissipative term, as will be shown in

Proposition 2.2 Let $U_{\infty}$ given as in theorem 2.1 if $\mathbf{u} \in \mathbb{R}^{2}$ satisfies $|\mathbf{u}| \leq U_{\infty}$, then $P(\mathbf{u}) \cdot \mathbf{u} \geq 0$.

We set

$$
\dot{H}^{m}\left(\mathbb{T}^{2}\right)=\left\{w \in H^{m}\left(\mathbb{T}^{2}\right), \quad \int w=0\right\}, m \geq 0
$$

and state

Theorem 2.3 Let $m \geq 3, u_{0} \in \dot{H}^{m}\left(\mathbb{T}^{2}\right)^{2}$. There exists $T_{m}>0, \delta_{m}>0$, such that: if $\left\|u_{0}\right\|_{L^{\infty}} \leq \delta_{m}$, (Int) has a unique strong solution

$$
u \in \mathcal{C}^{0}\left(\left[0, T_{m}\right] ; \dot{H}^{m}\right)^{2} \cap \mathcal{C}^{1}\left(\left(0, T_{m}\right] ; \dot{H}^{m-1}\right)^{2}
$$

Once these auxiliary systems are solved, we have the following convergence result

Theorem 2.4 Let $u_{0} \in \dot{H}^{5}\left(\mathbb{T}^{2}\right)^{2}$. Let $u$ the associate solution of (Int). We define $u^{0}$ on $\Omega^{\varepsilon}$ by

$$
u^{0}=\left(\begin{array}{l}
u \\
0
\end{array}\right) \text { in } \Omega, \quad u^{0}=0 \text { in } \Omega^{\varepsilon}-\Omega .
$$

There exists $\delta>0$ and $T>0$ such that, for any weak solution $u^{\varepsilon}$ of (1.3), (1.4), $(2.5)$

$$
\left(\sup \left|u_{0}\right| \leq \delta \text { and }\left\|\left(u^{\varepsilon}-u^{0}\right)(0, \cdot)\right\|_{L^{2}} \underset{\varepsilon \rightarrow 0}{\longrightarrow} 0\right) \quad \Longrightarrow \quad\left\|u^{\varepsilon}-u^{0}\right\|_{L^{\infty}\left(0, T ; L^{2}\right)} \underset{\varepsilon \rightarrow 0}{\longrightarrow} 0 .
$$

\section{Remarks:}

1. System (BL) is a generalization of the differential system satisfied by the Ekman profile in the case of flat boundaries:

$$
\mathbf{e} \times \tilde{u}-\frac{\partial^{2} \tilde{u}}{\partial Z^{2}}=0, \quad \tilde{u}(0)=-\left(\begin{array}{c}
\mathbf{u} \\
0
\end{array}\right) .
$$

As in the study of stationary Navier-Stokes equations, a smallness assumption on $|\mathbf{u}|$ is required to make the system (BL) well posed. Indeed, as pointed out in [24, 11], systems of this type may have two distinct solutions at large "Reynolds number": we refer to [11] for more details.

2. System (Int) is a generalization of the damped Euler equation satisfied by the interior term in the case of flat boundaries (see [15])

$$
\partial_{t} \zeta+u \cdot \nabla \zeta+\sqrt{2} \zeta=0, \quad \operatorname{div} u=0 .
$$

In this last system, the damping term $\sqrt{2} \zeta$ leads to a decrease of the $L^{\infty}$ norm of $\zeta$. As for 2D Euler equations (see [4, 17]), the method of Yudovitch applies and yields 
the existence of global smooth solutions.

For system (Int), we do not manage to get a so good control on operator $P$, so that we only have the existence of regular solutions for short times. Note that the smallness assumption on $\|u\|_{L^{\infty}}$ (or equivalently $\left\|u_{0}\right\|_{L^{\infty}}$ ) is linked to the solvability of (BL).

3. The proof of theorem 2.4 says more than the theorem itself: broadly speaking, it shows that as long as the solution $u$ of (Int) remains smooth and small in $L^{\infty}$ norm, there is convergence of weak solution $u^{\varepsilon}$ to $u$ (for appropriate initial data). With this formulation, we see that it is an extension of the convergence theorem stated in [7].

4. Proposition 2.2 is physically expected. It is a consequence of the so-called "Ekman pumping". The boundary layer creates an inflow and thus a circulation of fluid which increases energy dissipation. A numerical study of this pumping will be the matter of a forthcoming paper.

\section{Construction of an approximate solution}

\subsection{Ansatz}

We look for an Ansatz of type: for all $t>0$, for all $(x, y, z) \in \Omega^{\varepsilon}$,

$$
\begin{aligned}
& u_{a p p}^{\varepsilon}(t, x, y, z)=\sum_{i=0}^{n} \varepsilon^{i}\left(u^{i}(t, x, y, z)+\tilde{u}^{i}\left(t, x, y, \frac{x}{\varepsilon}, \frac{y}{\varepsilon}, \frac{z}{\varepsilon}\right)+\bar{u}^{i}\left(t, x, y, \frac{x}{\varepsilon}, \frac{y}{\varepsilon}, \frac{z-1}{\varepsilon}\right)\right), \\
& p_{\text {app }}^{\varepsilon}(t, x, y, z)=\sum_{i=0}^{n} \varepsilon^{i}\left(p^{i}(t, x, y, z)+\tilde{p}^{i}\left(t, x, y, \frac{x}{\varepsilon}, \frac{y}{\varepsilon}, \frac{z}{\varepsilon}\right)+\bar{p}^{i}\left(t, x, y, \frac{x}{\varepsilon}, \frac{y}{\varepsilon}, \frac{z-1}{\varepsilon}\right)\right) .
\end{aligned}
$$

- $u^{i}=u^{i}(t, x, y, z)\left(\right.$ resp. $\left.p^{i}=p^{i}(t, x, y, z)\right)$ is an interior term, 1-periodic in $(x, y)$, defined for $t \in \mathbb{R}^{+}$, and $(x, y, z) \in \Omega^{\varepsilon}$.

- $\tilde{u}^{i}=\tilde{u}^{i}(t, x, y, X, Y, Z)$ (resp. $\left.\tilde{p}^{i}=\tilde{p}^{i}(t, x, y, X, Y, Z)\right)$ is a lower boundary layer term, 1-periodic in $(x, y)$ and $(X, Y)$, defined for $t \in \mathbb{R}^{+},(x, y) \in(0,1)^{2}$, $(X, Y, Z) \in \tilde{\omega}$.

- $\bar{u}^{i}=\bar{u}^{i}(t, x, y, X, Y, Z)$ (resp. $\left.\bar{p}^{i}=\bar{p}^{i}(t, x, y, X, Y, Z)\right)$ is an upper boundary layer term, 1-periodic in $(x, y)$ and $(X, Y)$, defined for $t \in \mathbb{R}^{+},(x, y) \in(0,1)^{2}$, $(X, Y, Z) \in \bar{\omega}$.

It remains to add boundary conditions on these profiles. We impose that interior terms equal zero outside the interior domain $\Omega$ : for all $i$,

$$
\forall t \geq 0, \forall \mathbf{x} \in \Omega^{\varepsilon}-\Omega, \quad u^{i}(t, \mathbf{x})=0, \quad p^{i}(t, \mathbf{x})=0 .
$$

The boundary layer terms satisfy

$$
\tilde{u}^{i}=0 \text { on } \tilde{\gamma}, \quad \bar{u}^{i}=0 \text { on } \bar{\gamma} .
$$


and are expected to play no role outside the layer, which can be written

$$
\tilde{u}^{i}, \bar{u}^{i} \underset{Z \rightarrow \pm \infty}{\longrightarrow} 0, \quad \tilde{p}^{i}, \bar{p}^{i} \underset{Z \rightarrow \pm \infty}{\longrightarrow} 0 .
$$

We need further boundary conditions for the interfaces. The reason is the following: in order to prove the convergence of $u^{\varepsilon}$ to $u^{0}$, we need to carry energy estimates on $v^{\varepsilon}=u^{\varepsilon}-u_{\text {app }}^{\varepsilon}, q^{\varepsilon}=p^{\varepsilon}-p_{\text {app }}^{\varepsilon}$. In these estimates, treatment of pressure and viscosity terms leads to:

$$
\begin{aligned}
& \int \varepsilon^{-1} \nabla q^{\varepsilon} \cdot v^{\varepsilon}-\varepsilon \int \Delta v^{\varepsilon} \cdot v^{\varepsilon}= \\
&+\int\left|\nabla v^{\varepsilon}\right|^{2}+\int_{\Sigma_{1} \cup \Sigma_{2}}\left[\left(\varepsilon \frac{\partial u_{a p p}^{\varepsilon}}{\partial \mathbf{n}}-\varepsilon^{-1} p_{a p p}^{\varepsilon} \vec{n}\right) \cdot u_{a p p}^{\varepsilon}\right]_{\mid \Sigma_{1} \cup \Sigma_{2}} .
\end{aligned}
$$

We see that this last surface integral must be small enough, so that energy estimates allow to conclude. Note that it might not be the case if $u_{a p p}^{\varepsilon}$ was only including interior terms, because interior terms have a priori strong discontinuities at $\Sigma_{1} \cup \Sigma_{2}$ (and boundary layer terms are added to compensate these discontinuities). Sufficient conditions for this integral to vanish are the following jump conditions: $\forall(x, y) \in \mathbb{T}^{2}$,

$$
\left[\tilde{u}^{i}(t, x, y, \cdot)\right]_{\mid \Sigma}=-\left[u^{i}(t, x, y, \cdot)\right]_{\mid z=0}
$$

(which expresses a natural continuity condition of $u_{\text {app }}^{\varepsilon}$ at the interface $\Sigma_{1}$ ),

$$
\begin{gathered}
{\left[\tilde{p}^{0}(t, x, y, \cdot)\right]_{\mid \Sigma}=-\left[p^{0}(t, x, y, \cdot)\right]_{\mid z=0},} \\
{\left[\left(\frac{\partial \tilde{u}^{0}}{\partial Z}-\tilde{p}^{1} \mathbf{e}\right)(t, x, y, \cdot)\right]_{\mid \Sigma}=-\left[-p^{1}(t, x, y, \cdot) \mathbf{e}\right]_{\mid z=0},} \\
{\left[\left(\frac{\partial \tilde{u}^{i}}{\partial Z}-\tilde{p}^{i+1} \mathbf{e}\right)(t, x, y, \cdot)\right]_{\mid \Sigma}=-\left[\left(\frac{\partial u^{i-1}}{\partial z}-p^{i+1}\right)(t, x, y, \cdot) \mathbf{e}\right]_{\mid z=0}, \quad i \geq 1 .}
\end{gathered}
$$

Similar jump conditions hold of course for the upper boundary layer.

\subsection{Formal asymptotic expansion}

We do not detail the computations here, and refer to [12] for an exhaustive treatment.

- In the interior, $u^{0}$ satisfies the so-called "Taylor-Proudman theorem": it is invariant along the direction of the rotation axis. More precisely, we obtain here

$$
u^{0}=\left(\begin{array}{c}
u(t, x, y) \\
0
\end{array}\right)
$$

- In the boundary layer, variables $t, x$ and $y$ are simply parameters. If we set

$$
\mathbf{u}=u(t, x, y), \quad \tilde{u}=\left(\tilde{u}_{1}^{0}(t, x, y, \cdot), \tilde{u}_{2}^{0}(t, x, y, \cdot), \tilde{u}_{3}^{0}(t, x, y, \cdot)\right)^{t},
$$


one verifies that $\tilde{u}$ is formally solution of equations $(\mathrm{BL})$.

- Finally, we obtain the equation satisfied by $u(t, x, y)$. It is the $2 \mathrm{D}$ modified Euler equation (Int), with additional term $P(u)=\tilde{P}(u)+\bar{P}(u)$, where for instance, the bottom pumping term $\tilde{P}$ is (still formally) defined by:

$$
\text { For } \mathbf{u} \in \mathbb{R}^{2}, \quad \tilde{P}(\mathbf{u})=\int_{\tilde{\omega}}\left(\begin{array}{c}
\tilde{u}_{2} \\
-\tilde{u}_{1}
\end{array}\right), \quad \text { where } \tilde{u} \text { is solution of }(\mathrm{BL}) \text {. }
$$

Of course, the upper boudary layer term $\bar{u}$ and corresponding pumping term $\bar{P}$ are derived and defined exactly in the same way.

\subsection{The boundary layer system}

In this subsection, we give the main steps of the proof of theorem 2.1.

\section{Change of unknown}

We make a change of unknown function, so as to get jump conditions appropriate for a variational formulation. More precisely, let $\mathbf{u} \in \mathbb{R}^{2}$. Let $U_{E}$ be the "Ekman flow" defined by

$$
\begin{aligned}
& U_{E, 1}(X, Y, Z)=-e^{-Z \sqrt{2}}\left(u_{1} \cos \left(\frac{Z}{\sqrt{2}}\right)+u_{2} \sin \left(\frac{Z}{\sqrt{2}}\right)\right) \quad \text { in } \tilde{\omega}^{+}, \\
& U_{E, 2}(X, Y, Z)=-e^{-Z \sqrt{2}}\left(u_{2} \cos \left(\frac{Z}{\sqrt{2}}\right)-u_{1} \sin \left(\frac{Z}{\sqrt{2}}\right)\right) \quad \text { in } \tilde{\omega}^{+}, \\
& U_{E, 3}(X, Y, Z)=0 \quad \text { in } \tilde{\omega}^{+}, \quad U_{E}=0 \quad \text { in } \tilde{\omega}^{-}
\end{aligned}
$$

and let $\Pi_{E} \equiv 0$. It is known from $[20,15]$ that $\left(U_{E}, \Pi_{E}\right)$ is solution of the first two equations of (BL) in $\tilde{\omega}^{+}$. Setting $\tilde{u}=U_{E}+v$, the solvability of (BL) is equivalent to the solvability of

$$
\begin{aligned}
\mathbf{e} \times v+\nabla \tilde{p}+U_{E} \cdot \nabla+v \cdot \nabla U_{E}+v \cdot \nabla v-\Delta v=\left(\begin{array}{c}
-\mathbf{u}^{\perp} \\
0
\end{array}\right) & \text { in } \tilde{\omega}^{-}, \\
\mathbf{e} \times v+\nabla \tilde{p}+U_{E} \cdot \nabla+v \cdot \nabla U_{E}+v \cdot \nabla v-\Delta v=0 & \text { in } \tilde{\omega}^{+}, \\
\operatorname{div} v=0 & \text { in } \tilde{\omega}^{+} \cup \tilde{\omega}^{-}, \\
{[v]_{\mid \Sigma}=0 } & \text { at } \Sigma, \\
{\left[\frac{\partial v}{\partial Z}-\tilde{p} \mathbf{e}\right]_{\mid \Sigma}=-\left[\frac{\partial U_{E}}{\partial Z}\right]_{\mid \Sigma} } & \text { on } \Sigma, \\
v=0 \quad \text { on } \tilde{\gamma}, & v 1 \text { 1-periodic in }(X, Y) .
\end{aligned}
$$

\section{Variational formulation}

$$
\begin{aligned}
& \text { Let } \tilde{\Gamma}=\bigcup_{k \in \mathbb{Z}^{2}}(\tilde{\gamma}+(k, 0)), \tilde{\Omega}=\bigcup_{k \in \mathbb{Z}^{2}}(\tilde{\omega}+(k, 0)) \text {. Let } \\
& \qquad \mathcal{V}=\left\{\varphi \in \mathcal{C}^{\infty}(\tilde{\Omega})^{3}, \quad 1-\text { periodic in }(X, Y), \quad \operatorname{div} \varphi=0, \quad \operatorname{supp} \varphi \cap \tilde{\gamma}=\emptyset\right\},
\end{aligned}
$$




$$
V=\left\{v \in H_{l o c}^{1}(\tilde{\omega})^{3}, \quad \nabla v \in L^{2}(\tilde{\omega})^{9}, \quad v=0 \text { on } \tilde{\gamma}\right\}
$$

We denote $\|v\|_{V}=\left(\int_{\tilde{\omega}}|\nabla v|^{2}\right)^{1 / 2}$. We prove the existence of a variational solution of (BL2):

Theorem 3.1 There exists $U_{\infty}$ such that for $|\mathbf{u}| \leq U_{\infty}$, (BLD) has a solution: more precisely, there exists $v \in V$ such that $: \forall \varphi \in \mathcal{V}$,

$$
\begin{aligned}
& \int_{\tilde{\omega}}(\mathbf{e} \times v) \cdot \varphi+\int_{\tilde{\omega}}\left(U_{E} \cdot \nabla v\right) \cdot \varphi+\int_{\tilde{\omega}}\left(v \cdot \nabla U_{E}\right) \cdot \varphi+ \\
& \int_{\tilde{\omega}}(v \cdot \nabla v) \cdot \varphi+\int_{\tilde{\omega}} \nabla v \cdot \nabla \varphi=\left(\begin{array}{c}
-\mathbf{u}^{\perp} \\
0
\end{array}\right) \cdot \int_{\tilde{\omega}^{-}} \varphi+\left[\frac{\partial U_{E}}{\partial Z}\right]_{\mid \Sigma} \cdot \int_{\Sigma} \varphi .
\end{aligned}
$$

Proof: The proof uses classical Galerkin approximation. As for the classical NavierStokes equation, the smallness condition on $|\mathbf{u}|$ allows to prove the existence of a solution to each finite dimensional approximate problem, through the use of Brouwer theorem. We refer to [12] for all details. Note that the space of test functions has compact support. As we still do not know the behavior at infinity of a solution, we can not test it in the variational formulation, as it would be the case in bounded domains. In particular, at this stage of the proof, we do not know if there is only one solution.

\section{De St-Venant estimates}

The main step is to show the exponential decrease of the boundary layer term, and estimate (2.6). We consider separately the oscillatory part, for which we have all the desired Sobolev injections, and the horizontal average of a solution $\tilde{u}$ on $\tilde{\omega}^{+}$. Thus, to all $w \in L_{\text {loc }}^{1}\left(\mathbb{T}^{2} \times \mathbb{R}^{+}\right)^{N}(N \geq 1)$, we associate its average $\langle w\rangle \in L_{\text {loc }}^{1}\left(\mathbb{R}^{+}\right)^{N}$ and its oscillatory part $w^{*} \in L_{l o c}^{1}\left(\mathbb{T}^{2} \times \mathbb{R}^{+}\right)^{N}$. Namely,

$$
\forall \mathbf{X}=(X, Y, Z) \in \mathbb{T}^{2} \times \mathbb{R}^{+}, \quad\langle w\rangle(Z)=\int_{\mathbb{T}^{2} \times\{Z\}} w d X d Y, \quad w^{*}(\mathbf{X})=w(\mathbf{X})-\langle w\rangle(Z) .
$$

Note that we have:

$$
\nabla w \in L^{2}\left(\tilde{\omega}^{+}\right)^{9} \Longrightarrow w^{*} \in H^{1}\left(\tilde{\omega}^{+}\right)^{3}
$$

- First step : Average and Oscillations.

One verifies that $\left\langle u_{3}\right\rangle=0$. Then, let $V=\left\langle\tilde{u}_{1}\right\rangle+i\left\langle\tilde{u}_{2}\right\rangle, \quad F=\left\langle\left(\tilde{u}^{*} \cdot \nabla \tilde{u}^{*}\right)_{1}\right\rangle+$ $i\left\langle\left(\tilde{u}^{*} . \nabla \tilde{u}^{*}\right)_{2}\right\rangle, \quad i^{2}=-1$. One verifies that

$$
i V-V^{\prime \prime}=F \text {. }
$$

Equation (3.18) is a simple linear diferential equation. This allows us to have precise information on $\langle\tilde{u}\rangle$ relatively to the right member $\left\langle\left(\tilde{u}^{*} . \nabla \tilde{u}^{*}\right)\right\rangle$. We get

Proposition 3.2 There exist $C>0, \sigma>0$, such that for all $R>0$,

$$
\|\tilde{u}\|_{H^{1}\left(\tilde{\omega}^{R}\right)} \leq C\left(\exp (-\sigma R)+\left\|\nabla \tilde{u}^{*}\right\|_{L^{2}\left(\tilde{\omega}^{R / 2}\right)}\right) .
$$


- Second step : Higher order derivatives

We control for all $m$ the norms $H^{m}\left(\tilde{\omega}^{R+1}\right)$ of $\tilde{u}, \tilde{p}$ by the mean of the norm $H^{1}\left(\tilde{\omega}^{R}\right)$ of $\tilde{u}$. More precisely,

Proposition 3.3 For all $R>0$, for all $m \geq 0,\left(u^{*}, \tilde{p}^{*}\right) \in H^{m}\left(\tilde{\omega}^{R+1}\right)^{4}$ with estimate

$$
\|\tilde{u}\|_{H^{m}\left(\tilde{\omega}^{R+1}\right)}+\|\nabla \tilde{p}\|_{H^{m}\left(\tilde{\omega}^{R+1}\right)} \leq C_{m}\|\tilde{u}\|_{H^{1}\left(\tilde{\omega}^{R}\right)}
$$

Proof: It uses regularity results due to the ellipticity of the Stokes operator. The key point is that one may control the $W^{m+2, q}$ norm in a strip by the $W^{m, q}$ norm in a slighty larger strip. Precisely,

Lemma 3.4 Let $u, \tau, f \in \mathcal{C}^{\infty}\left(\tilde{\omega}^{+}\right)$solution of

$$
\begin{aligned}
\Delta u=\nabla \tau+f & \text { in } \tilde{\omega}^{+}, \\
\nabla \cdot u=0 & \text { in } \tilde{\omega}^{+}, \\
u \quad 1-\text { periodic } & \text { in }(X, Y) .
\end{aligned}
$$

Then, for all $s \geq 1, \delta \in(0, s)$, for all $m \geq 0$ and $q \geq 1$,

$$
\|u\|_{m+2, q, s, s+1}+\|\nabla \tau\|_{m, q, s, s+1} \leq C\left(\|f\|_{m, q, s-\delta, s+1+\delta}+\|u\|_{1, q, s-\delta, s+1+\delta}\right),
$$

$C$ being independent of $s$.

- Third step :

Thanks to former steps, it remains to show the decay of the function $f(R)=$ $\left\|\nabla \tilde{u}^{*}\right\|_{L^{2}\left(\tilde{\omega}^{R}\right)}$, as a function of $R$. Through energy estimates (cf [12]), one obtains, for $R$ large enough, the following integro-differential inequality

$$
f^{\prime}(R)+a \int_{R}^{+\infty} f(R) \leq b f(R), \quad a, b>0 .
$$

Thanks to a Gronwall type lemma, we conclude to

Proposition 3.5 There exists $R_{1}, C, \sigma>0$ such that

$$
\forall R \geq R_{1}, \quad\left\|\nabla \tilde{u}^{*}\right\|_{L^{2}\left(\tilde{\omega}^{R}\right)} \leq C \exp (-\sigma R) .
$$

Once this exponential decay is obtained, we conclude to the unicity of the solution by classical arguments.

\subsection{The interior system}

Let us take $U_{\infty}$ as given in theorem 2.1. We may now rigorously define

$$
\forall \mathbf{u} \in \mathbb{R}^{2}, \quad|\mathbf{u}| \leq U_{\infty}, \quad \tilde{P}(\mathbf{u})=\int_{\tilde{\omega}}\left(\begin{array}{c}
\tilde{u}_{2} \\
-\tilde{u}_{1}
\end{array}\right) d \mathbf{X}
$$

where $\tilde{u}$ is solution of (BL) (similaly, we define $\bar{P}$, and then $P=\tilde{P}+\bar{P}$ ). Operator $P$ is dissipative, as stated in proposition 2.2. Indeed, it is shown in [12] that 


$$
\tilde{P}(\mathbf{u}) \cdot \mathbf{u}=\int_{\tilde{\omega}}|\nabla \tilde{v}|^{2} \geq 0,
$$

where $\tilde{v}$ is defined on $\tilde{\omega}$ by

$$
\tilde{v}=\tilde{u} \text { on } \tilde{\omega}^{+}, \quad \tilde{v}=\tilde{u}-\left(\begin{array}{c}
\mathbf{u} \\
0
\end{array}\right) \text { on } \tilde{\omega}^{-} .
$$

The proof of the existence and unicity of a regular solution for (Int) relies on a standard iterative scheme. Obviously, the keypoint is to have a good estimate on $P(u)$ in $L^{\infty}\left(0, T ; H^{m}\right)$. This is done up to a sharper control on sup $|u|$, as stated in

Proposition 3.6 For all $m \geq 3$, there exists $\delta_{m}>0$, and an increasing function $\varphi_{m} \in C\left(\mathbb{R}^{+}, \mathbb{R}^{+}\right)$, such that, for all $T>0$ and $u \in L^{\infty}\left(0, T ; H^{m}\left(\mathbb{T}^{2}\right)\right)^{2}$,

$$
\sup |u| \leq \delta_{m} \quad \Longrightarrow \quad\|\tilde{P}(u)\|_{L^{\infty}\left(H^{m}\right)} \leq \varphi_{m}\left(\|u\|_{L^{\infty}\left(H^{m}\right)}\right) .
$$

Similarly, we obtain

Proposition 3.7 For all $m \geq 3$, there exists $\varphi_{m} \in C\left(\mathbb{R}^{+} \times \mathbb{R}^{+}, \mathbb{R}^{+}\right)$and $\delta_{m}>0$, such that, for all $T>0$ and for all $u, u^{\prime} \in L^{\infty}\left(0, T, H^{m}\right)^{2}, \sup |u|+\sup \left|u^{\prime}\right| \leq \delta_{m}$ implies

$$
\left\|\tilde{P}(u)-\tilde{P}\left(u^{\prime}\right)\right\|_{L^{\infty}\left(H^{m}\right)} \leq \varphi_{m}\left(\|u\|_{L^{\infty}\left(H^{m}\right)},\left\|u^{\prime}\right\|_{L^{\infty}\left(H^{m}\right)}\right)\left\|u-u^{\prime}\right\|_{L^{\infty}\left(H^{m}\right)}
$$

\section{Final Energy estimates}

On the basis of the analysis led in previous section, we show the existence of an approximate solution $u_{a p p}^{\varepsilon}, p_{a p p}^{\varepsilon}$, of (1.3), (1.4). It is built in such a way that $w^{\varepsilon}=$ $u^{\varepsilon}-u_{\text {app }}^{\varepsilon}$ satisfies

$$
\begin{aligned}
\partial_{t} w^{\varepsilon}+\frac{\mathbf{e} \times w^{\varepsilon}}{\varepsilon}+\frac{\nabla q^{\varepsilon}}{\varepsilon}-\varepsilon \Delta w^{\varepsilon}+w^{\varepsilon} \cdot \nabla u_{a p p}^{\varepsilon}+u^{\varepsilon} \cdot \nabla w^{\varepsilon} & =\tilde{R}^{\varepsilon} \text { in } \Omega \cup \Omega_{i}^{\varepsilon}, \\
\nabla \cdot w^{\varepsilon} & =0 \text { in } \Omega \cup \Omega_{i}^{\varepsilon}, \\
{\left[w^{\varepsilon}\right]_{\mid \Sigma_{i}} } & =0 \text { at } \Sigma_{i}, \\
{\left[\varepsilon \frac{\partial w^{\varepsilon}}{\partial n}-\varepsilon^{-1} q^{\varepsilon} \vec{n}\right]_{\mid \Sigma_{i}} } & =\tilde{\sigma}^{\varepsilon} \text { at } \Sigma_{i}, \\
w^{\varepsilon} & =0 \text { at } \Gamma_{1}^{\varepsilon} \cup \Gamma_{2}^{\varepsilon}
\end{aligned}
$$

with

$$
\begin{gathered}
\tilde{R}^{\varepsilon}=R_{1}^{\varepsilon}+R_{2}^{\varepsilon}, \quad\left\|R_{1}^{\varepsilon}\right\|_{L^{\infty}\left(L^{2}\right)}=O\left(\varepsilon^{1 / 2}\right), \\
\left\|R_{2}^{\varepsilon}\right\|_{L^{\infty}\left(H^{-1}\right)}=O\left(\varepsilon^{3 / 2}\right), \quad\left\|\tilde{\sigma}^{\varepsilon}\right\|_{L^{\infty}\left(H^{-1 / 2}\right)}=O(\varepsilon) .
\end{gathered}
$$

In the energy estimates on $w^{\varepsilon}$, the term $\int_{\Omega^{\varepsilon}}\left(w^{\varepsilon} \cdot \nabla u_{\text {app }}^{\varepsilon}\right) \cdot w^{\varepsilon}$ is the hardest to control, because the boundary layer part of the approximate solution has strong gradients. Focusing as usual on the bottom layer, the worst term is then

$$
J^{\varepsilon}=\varepsilon^{-1} \int_{\Omega^{\varepsilon}}\left(w^{\varepsilon} \cdot \nabla_{\mathbf{X}} \tilde{u}^{0}\left(x, y, \frac{x}{\varepsilon}, \frac{y}{\varepsilon}, \frac{z}{\varepsilon}\right)\right) w^{\varepsilon} .
$$


Introducing the auxiliary function

$$
G(X, Y, Z)=\sup _{x, y \in \mathbb{T}^{2}}\left|\nabla_{X} \tilde{u}^{0}(x, y, X, Y, Z)\right|
$$

we have

$$
\left|J^{\varepsilon}\right| \leq \varepsilon^{-1} \int_{\Omega^{\varepsilon}}\left|w^{\varepsilon}\right|^{2} G\left(\frac{x}{\varepsilon}, \frac{y}{\varepsilon}, \frac{z}{\varepsilon}\right) d \mathbf{x} .
$$

We are left with the control of

$$
\begin{aligned}
I_{\varepsilon}=\int_{\Omega^{\varepsilon}}\left|w^{\varepsilon}\right|^{2} G\left(\frac{x}{\varepsilon}, \frac{y}{\varepsilon}, \frac{z}{\varepsilon}\right) d \mathbf{x} & =\int_{\Omega^{\varepsilon} \cap\{z>R \varepsilon\}}\left|w^{\varepsilon}\right|^{2} G\left(\frac{x}{\varepsilon}, \frac{y}{\varepsilon}, \frac{z}{\varepsilon}\right) d \mathbf{x} \\
& +\int_{\Omega^{\varepsilon} \cap\{z<R \varepsilon\}}\left|w^{\varepsilon}\right|^{2} G\left(\frac{x}{\varepsilon}, \frac{y}{\varepsilon}, \frac{z}{\varepsilon}\right) d \mathbf{x}=I_{R}^{\varepsilon,+}+I_{R}^{\varepsilon,-},
\end{aligned}
$$

where $R>0$ will be chosen below.

Study of $I_{R}^{\varepsilon,+}$ : Thanks to proposition 3.3, there exists $R_{1}, C, \sigma>0$ such that for all $Z>R_{1}$,

$$
G(X, Y, Z) \leq C \exp (-\sigma Z) .
$$

Hence, for all $\delta>0$, there exists $R=R(\delta)>R_{1}$ such that

$$
\sup _{X, Y, Z \geq R} Z^{2} G(X, Y, Z) \leq \delta .
$$

Then we may write

$$
\begin{aligned}
I_{R}^{\varepsilon,+} & \leq \varepsilon^{2}\left(\sup _{X, Y, Z \geq R} Z^{2} G(X, Y, Z)\right) \int_{\Omega^{\varepsilon} \cap\{z>R \varepsilon\}}\left|\frac{w^{\varepsilon}}{z}\right|^{2} \\
& \leq C \delta \varepsilon^{2}\left\|\nabla w^{\varepsilon}\right\|_{L^{2}}^{2}
\end{aligned}
$$

where we have used Hardy's inequality. Chosing $\delta=1 / 4 C$ and $R=R(\delta)$, it leads to

$$
I_{R}^{\varepsilon,+} \leq \frac{\varepsilon^{2}}{4}\left\|\nabla w^{\varepsilon}\right\|_{L^{2}}^{2} .
$$

Study of $I_{R}^{\varepsilon,-}$ : It remains to bound

$$
I_{R}^{\varepsilon,-}=\int_{\Omega^{\varepsilon} \cap\{z \leq R \varepsilon\}} w^{\varepsilon}(x, y, z)^{2} G\left(\frac{x}{\varepsilon}, \frac{y}{\varepsilon}, \frac{z}{\varepsilon}\right) d x d y d z .
$$

We can write

$$
\Omega^{\varepsilon} \cap\{z \leq R \varepsilon\}=\bigcup_{k \in K \subset \mathbb{Z}^{2}} \varepsilon(\omega+k), \quad \operatorname{card}(K)=\frac{n}{\varepsilon^{2}}
$$

for a domain $\omega$. Now,

$$
\begin{aligned}
I_{R}^{\varepsilon,-} & =\varepsilon^{3} \sum_{k \in K} \int_{\omega+k}\left|w^{\varepsilon}(\varepsilon X, \varepsilon Y, \varepsilon Z)\right|^{2} G(X, Y, Z) d X d Y d Z \\
& \leq \varepsilon^{3} \sum_{k \in K}\|G\|_{L^{2}(\omega+k)}\left(\int_{\omega+k}\left|w^{\varepsilon}(\varepsilon X, \varepsilon Y, \varepsilon Z)\right|^{4} d X d Y d Z\right)^{1 / 2} .
\end{aligned}
$$


As $F$ is periodic

$$
I_{R}^{\varepsilon,-} \leq \varepsilon^{3}\|G\|_{L^{2}(\omega)} \sum_{k \in K}\|\tilde{w}\|_{L^{4}(\omega+k)}^{2}
$$

where $\tilde{w}(X, Y, Z)=w^{\varepsilon}(\varepsilon X, \varepsilon Y, \varepsilon Z)$. By Sobolev imbedding

$$
\|\tilde{w}\|_{L^{4}(\omega+k)}^{2} \leq C\|\nabla \tilde{w}\|_{H^{1}(\omega+k)}^{2}
$$

where $C$ is independent of $k$. So

$$
\begin{aligned}
I_{R}^{\varepsilon,-} & \leq C \varepsilon^{3}\|G\|_{L^{2}(\tilde{\omega})} \int_{\cup(\omega+k)}\left|\nabla_{\mathbf{X}} \tilde{w}(X, Y, Z)\right|^{2} d X d Y d Z \\
& \leq C \varepsilon^{2}\|G\|_{L^{2}(\tilde{\omega})}\left\|\nabla w^{\varepsilon}\right\|_{L^{2}\left(\Omega^{\varepsilon}\right)}^{2}
\end{aligned}
$$

We thus have

$$
\begin{aligned}
I_{R}^{\varepsilon,-} & \leq C^{\prime} \varepsilon^{2} \sup _{x, y \in \mathbb{T}^{2}}\left\|\nabla \tilde{u}^{0}\right\|_{L^{2}(\tilde{\omega})}\left\|\nabla w^{\varepsilon}(t, \cdot)\right\|_{L^{2}}^{2} \\
& \leq C^{\prime \prime} \varepsilon^{2}\left(\sup \left|u^{0}\right|\right)\left\|\nabla w^{\varepsilon}(t, \cdot)\right\|_{L^{2}}^{2},
\end{aligned}
$$

Then, assuming that $\sup \left|u^{0}\right|$ is small, it yields

$$
I_{R}^{\varepsilon,-} \leq \frac{\varepsilon^{2}}{4}\left\|\nabla w^{\varepsilon}(t, \cdot)\right\|_{L^{2}}^{2}
$$

Conclusion: Under a smallness assumption on $\sup \left|u^{0}\right|$, (which if fulfilled under a smallness assumption on $\left.\left\|u_{0}\right\|_{L^{\infty}}\right)$ we can absorb the squares of the $L^{2}$ norm of $\nabla w^{\varepsilon}$ at the right-hand side of (2.6) into the diffusive term $\varepsilon\left\|\nabla w^{\varepsilon}(t, \cdot)\right\|^{2}$ at the left-hand side. We find inequality of type

$$
\partial_{t}\left\|w^{\varepsilon}(t, \cdot)\right\|_{L^{2}}^{2}+\frac{\varepsilon}{2}\left\|\nabla w^{\varepsilon}(t, \cdot)\right\|_{L^{2}}^{2} \leq C(\varepsilon)+D\left\|w^{\varepsilon}(t, \cdot)\right\|_{L^{2}}^{2}
$$

with $C(\varepsilon) \rightarrow 0$ as $\varepsilon \rightarrow 0$. We conclude thanks to a Gronwall lemma.

\section{Remarks:}

1. Following [7], the convergence result may be refined: for arbitrary $s \in \mathbb{N}$, one can construct an approximate solution $u_{a p p, s}^{\varepsilon}$ of $(1.3),(1.4)$, in such a way that

$$
\left(u^{\varepsilon}-u_{a p p, s}^{\varepsilon}\right) \underset{\varepsilon \rightarrow 0}{\longrightarrow} 0, \quad \text { in } L^{\infty}\left(0, T ; H^{s}(\Omega)\right)^{3} .
$$

2. We have considered rough boundaries of type $\Gamma^{\varepsilon}:=\varepsilon \Gamma\left(\varepsilon^{-1} x, \varepsilon^{-1} y\right)$. We could have considered more general boundaries of type

$$
\Gamma^{\varepsilon}:=\varepsilon \Gamma\left(x, y, \varepsilon^{-1} x, \varepsilon^{-1} y\right)
$$




\section{References}

[1] Achdou, Y., Pironneau, O., and Valentin, F. Effective boundary conditions for laminar flows over periodic rough boundaries. J. Comput. Phys. 147, 1 (1998), 187-218.

[2] Amirat, Y., Bresch, D., Lemoine, J., And Simon, J. Effect of rugosity on a flow governed by navier-stokes equations. Quarterly Appl. Math 59, 4 (2001), 769-785.

[3] Bresch, D., Desjardins, D., And GÉrard-Varet, D. Rotating fluids in a cylinder. Prepublication UMPA 317, 2003.

[4] Chemin, J.-Y. Perfect incompressible fluids, vol. 14 of Oxford Lecture Series in Mathematics and its Applications. The Clarendon Press Oxford University Press, New York, 1998. Translated from the 1995 French original by Isabelle Gallagher and Dragos Iftimie.

[5] Chemin, J.-Y., Desjardins, B., Gallagher, I., And Grenier, E. Ekman boundary layers in rotating fluids. ESAIM Controle optimal et calcul des variations (2002).

[6] Colin, T., And Fabrie, P. Rotating fluid at high Rossby number driven by a surface stress: existence and convergence. Adv. Differential Equations 2, 5 (1997), 715-751.

[7] Desjardins, B., Dormy, E., And Grenier, E. Stability of mixed EkmanHartmann boundary layers. Nonlinearity 12, 2 (1999), 181-199.

[8] Desjardins, B., And Grenier, E. Derivation of quasi-geostrophic potential vorticity equations. Adv. Differential Equations 3, 5 (1998), 715-752.

[9] Dormy, E. Modélisation Numérique de la Dynamo Terrestre. PhD thesis, Institut de Physique du Globe de Paris, 1997.

[10] Espedal, M. S., Fasano, A., And Mikelić, A. Filtration in porous media and industrial application, vol. 1734 of Lecture Notes in Mathematics. SpringerVerlag, Berlin, 2000. Lectures from the 4th C.I.M.E. Session held in Cetraro, August 24-29, 1998, Edited by Fasano, Fondazione C.I.M.E.. [C.I.M.E. Foundation].

[11] Galdi, G. P. An introduction to the mathematical theory of the Navier-Stokes equations. Vol. II, vol. 39 of Springer Tracts in Natural Philosophy. SpringerVerlag, New York, 1994. Nonlinear steady problems.

[12] GÉrard-Varet, D. Highly rotating fluids in rough domains. Prepublication UMPA 314, 2003.

[13] Gerard-Varet, D., And Grenier, E. A zoology of boundary layers. Revista de la Real Academia de Ciencias 96, 3 (2002), 401-411. 
[14] Greenspan, H. The Theory of Rotating Fluids. Breukelen Press, 1968.

[15] Grenier, E., And Masmoudi, N. Ekman layers of rotating fluids, the case of well prepared initial data. Comm. Partial Differential Equations 22, 5-6 (1997), 953-975.

[16] JÄGER, W., AND Mikelić, A. On the roughness-induced effective boundary conditions for an incompressible viscous flow. J. Differential Equations 170, 1 (2001), 96-122.

[17] Marchioro, C., And Pulvirenti, M. Mathematical Theory of Incompressible Nonviscous Fluids. Springer Verlag, 1994.

[18] Masmoudi, N. Ekman layers of rotating fluids: the case of general initial data. Comm. Pure Appl. Math. 53, 4 (2000), 432-483.

[19] Narteau, C., Le Mouël, J.-L., J.-P. Poirier, J.-P., Sepulveda, E., And Shnirman, M. On a small scale roughness of the core-mantle boundary. Earth and Plan. Science Letters 191 (2001), 49-61.

[20] Pedlosky, J. Geophysical Fluid Dynamics. Springer Verlag, 1979.

[21] Rousset, F. Stability of large ekman layers in rotating fluids. submitted, 2003.

[22] Stewartson, K. On almost rigid rotations. J. Fluid Mech. 3 (1957), 17-26.

[23] Stewartson, K. On almost rigid rotations. part2. J. Fluid Mech. 26 (1965), $131-152$.

[24] Temam, R. Navier-Stokes Equations. North-Holland, 1985.

U.M.P.A, U.M.R. 5669, E.N.S. LYON, 46 Allée D'Italie, 69364 LyON CEDEX 07

David.GERARD-VARET@umpa.ens-lyon.fr www.umpa.ens-lyon.fr $/ \sim$ dgerardv 